\title{
Abundances on the main sequence of the globular cluster $\omega$ Centauri
}

\author{
G. S. Da Costa ${ }^{1}$, Laura M. Stanford ${ }^{1}$, John E. Norris ${ }^{1}$, \\ and R. D. Cannon ${ }^{2}$ \\ ${ }^{1}$ Research School of Astronomy \& Astrophysics, The Australian National Observatory \\ email: gdc, stanford, jen@mso.anu.edu.au \\ ${ }^{2}$ Anglo-Australian Observatory \\ email: rdc@aaoepp.aao.gov.au
}

\begin{abstract}
Using the 2dF multi-fibre instrument on the Anglo-Australian Telescope, moderate resolution spectra have been obtained for a large sample of stars on the main sequence and at the turnoff in the unusual globular cluster $\omega$ Cen. We investigate the behaviour of $\mathrm{CH}, \mathrm{CN}$ and SrII line strength indices as a function of overall abundance for the main sequence sample. A number of stars do not follow the relations defined by the majority. These anomalous objects can be categorized into (at least) three types. (1) Carbon enhanced stars, which represent about $5 \%$ of the sample, and which are found at all metallicities. Spectrum synthesis calculations show that the atmospheres of these stars are typically enhanced in carbon by factors of between 3 and 10. (2) Nitrogen enhanced stars, revealed for $[\mathrm{Fe} / \mathrm{H}] \geqslant-1.3$ by strong $\mathrm{CN}$ indices, which make up $\sim 40 \%$ of the cluster main sequence population above this metallicity. The stars are enhanced in nitrogen by factors of up to 100 . Our data, however, provide no constraints on their relative numbers at lower $[\mathrm{Fe} / \mathrm{H}]$. (3) Stars with enhancements of the s-process element Sr by factors of 30 to 60 . The possible origins for these abundance anomalies are discussed.
\end{abstract}

Keywords. Globular clusters: individual ( $\omega$ Cen), stars: abundances, stars: AGB and postAGB, stars: chemically peculiar

\section{Introduction}

The globular cluster $\omega$ Centauri has been recognised as an unusual object for at least three decades. Foremost among its peculiar characteristics is the large abundance range exhibited by the cluster stars: from $[\mathrm{Fe} / \mathrm{H}] \approx-1.8$ to least $[\mathrm{Fe} / \mathrm{H}] \approx-0.5$ (e.g. Pancino et al. 2002, and the references therein). Such a large abundance range is not observed in any other globular cluster. There is also evidence that the star formation in $\omega$ Cen took place over an extended period. This contention is based on both spectroscopic and photometric analyses. For example, high dispersion studies (e.g. Norris \& Da Costa 1995b, Smith et al. 2000) have revealed the chemical enrichment signatures of low mass $\left(\sim 1.5-3 \mathrm{M}_{\odot}\right)$ AGB stars in the cluster. Such stars have lifetimes of 1-3 Gyr. Similarly, population modelling of the horizontal branch morphology by Rey et al. (2004) suggests an age difference of perhaps 4 Gyr between the dominant metal-poor population and the most metal-rich stars. Hilker et al. (2004) reach a similar conclusion from their combined spectroscopic and photometric study. Indeed, it is now increasingly evident that the cluster contains a number of distinct populations, each with their own kinematic and chemical signatures (e.g. Norris et al. 1997, Sollima et al. 2005).

We are also studying the abundance and age distribution of the stars in this cluster through the use of photometry and moderate resolution spectroscopy of a large sample of cluster members on the main sequence and at the cluster turnoff. The results of that work will be presented elsewhere (Stanford et al. 2005a, in prep). In this contribution 
we concentrate on what can be learned from the stars in our samples that do not follow the relations exhibited by the majority of cluster members. In particular, among the bright red giants in $\omega$ Cen there are a number of objects with anomalous abundances, including stars with excesses of s-process elements (e.g. Lloyd-Evans 1983, Vanture et al. 2002) and CH-stars with strong overabundances of carbon (e.g. Bell \& Dickens 1974). We investigate here the occurrence of similar anomalous stars on the main sequence, and discuss their possible origins. A complete description of the results will be given in Stanford et al. (2005b, in prep).

\section{Observations}

The stars observed were drawn from a photometric catalogue generated from CCD images obtained with the $1 \mathrm{~m}$ telescope at Siding Spring Observatory. The catalogue contains $B, V$ photometry for ten slightly overlapping $20^{\prime} \times 20^{\prime}$ fields, centered $\sim 20^{\prime}$ from the cluster centre, that surround the cluster. The particular targets for the spectroscopic observations consist of uncrowded (no neighbours within $5^{\prime \prime}$ ) stars in two magnitude ranges, a main sequence sample with $18.0 \leqslant V \leqslant 18.5$ and $0.40 \leqslant B-V \leqslant 1.10$, and a turnoff sample with $17.25 \leqslant V \leqslant 18.0$ and $0.60 \leqslant B-V \leqslant 1.10$. The candidates were observed with the $2 \mathrm{dF}$ multi-fibre instrument on the Anglo-Australian Telescope. The spectrograph setup employed gave useful coverage of $\sim 900 \AA$ centred at $4200 \AA$, with a resolution of $\sim 2.5 \AA$ (FWHM). The availability of on-line data reduction software, and the high radial velocity of $\omega$ Cen $\left(232 \mathrm{kms}^{-1}\right)$ meant that shortly after each observation, stars could be classified as either cluster members or non-members. Non-member stars were replaced with new candidates and member stars were reobserved to build-up the signal-to-noise of their spectra. The final data set for the main sequence sample consists of spectra for just over 200 cluster members with typical S/N of $\sim 35$ at the G-band.

\section{Line Strength Indices}

\subsection{Metallicities}

The likely existence of an age-abundance relation in this cluster (e.g. Rey et al. 2004, Hilker et al. 2004) means that the determination of metal abundances for the observed stars by simple techniques, such as a comparison of line strengths with those of similar main sequence stars in clusters of known abundance, is not appropriate. This will particularly be the case for metal-rich $\omega$ Cen stars which are younger and therefore hotter, bluer and of weaker K-line strength than stars of similar $\mathrm{M}_{V}$ and abundance in standard clusters. To overcome this situation we have used the approach developed by Beers et al. (1999), in which the derived abundance, denoted by $[\mathrm{Fe} / \mathrm{H}]$, is determined from appropriate combinations of the equivalent width of the CaII K-line, the colour index $(B-V)_{0}$ and the ACF index, which is the autocorrelation of the spectrum over the region 4000$4280 \AA$ excluding the $\mathrm{H} \delta$ line and the CN-bands in the vicinity of $4215 \AA$. Consideration of the errors in these quantities indicates that the $[\mathrm{Fe} / \mathrm{H}]$ values have a typical uncertainty of \pm 0.2 dex. In the following we concentrate solely on the main sequence sample as it is relatively unbiased with respect to $(B-V)$ colour.

\section{2. $G$-band $(\mathrm{CH})$}

To measure the strength of the $\mathrm{CH}$ features we have computed the index $\mathrm{W}(\mathrm{G})$, which is a measure of the strength of the $\mathrm{G}$-band $(\mathrm{CH})$ at $\sim 4300 \AA$. The values of $\mathrm{W}(\mathrm{G})$ are plotted against $[\mathrm{Fe} / \mathrm{H}]$ in the left panel of Fig. 1. Shown also are the mean $W(G)$ values for similar sets of main sequence stars, observed with the same instrumental setup, in the standard 
globular clusters NGC $6397([\mathrm{Fe} / \mathrm{H}]=-1.95)$, NGC $6752(-1.56)$ and 47 Tuc $(-0.76)$. In 47 Tuc the main sequence stars possess an anti-correlation between the strengths of $\mathrm{CN}$ and $\mathrm{CH}$ features, and the stars can be classified as either $\mathrm{CN}$-strong ( $\mathrm{CH}$-weak) or $\mathrm{CN}$ weak (CH-strong). The two groups differ in carbon abundance with the CN-strong stars being depleted by $\sim 0.3$ dex relative to the $\mathrm{CN}$-weak stars (cf. Cannon et al. 1998, Briley et al. 2004). Consequently, there are two 47 Tuc points in the figure, representing the two populations. The $\omega$ Cen stars show a general increase in the $W(G)$ values with increasing metallicity. For the majority, the scatter in the $\mathrm{W}(\mathrm{G})$ indices is largely driven by errors in the measured quantities, particularly at the metal-poor end. For the more metal-rich stars, however, there are indications of an intrinsic spread in the $W(G)$ values, with some stars showing potentially significant $\mathrm{C}$ depletions similar to those for the 47 Tuc CN-strong stars.

Of particular interest are the 13 stars (plotted as filled stars in Fig. 1) that lie above the relation defined by the majority of the stars - these stars have larger $W(G)$ values for their $[\mathrm{Fe} / \mathrm{H}]$ than a 'typical' $\omega$ Cen main sequence member. It is immediately apparent from Fig. 1 that the 'strong G-band' stars are found at all metallicities: there is no indication that any particular $[\mathrm{Fe} / \mathrm{H}]$ is favoured over any other. Given the total sample size of 205 stars, the frequency of occurrence of these strong G-band main sequence stars is of order $5 \%$, making them relatively rare objects.

To investigate the atmospheric abundances implied by the strong G-bands, we have carried out spectrum synthesis calculations. The calculations assume our determination of $[\mathrm{Fe} / \mathrm{H}]$ for each star and use temperatures derived from the photometry. In each case the carbon abundance was varied until a satisfactory match was achieved between the observed and calculated spectra. We find that these strong G-band stars are typically enhanced in carbon by factors of $\sim 3$ to 10 , occasionally more, relative to the 'normal' $\omega$ Cen stars of comparable metallicity. We also find that these stars do not appear to require significant enhancements (or depletions) in $\mathrm{N}$, though we are sensitive to $\mathrm{N}$ abundance changes only for metallicities above $[\mathrm{Fe} / \mathrm{H}] \approx-1.3 \mathrm{dex}$.

\section{3. $C N-$ bands}

In the right panel of Fig. 1 we show a plot of the cyanogen index $\mathrm{S}(3839)$ against $[\mathrm{Fe} / \mathrm{H}]$. This index measures the strength of the CN bands at $3883 \AA$. The panel also shows mean points for the three standard clusters, with the points for 47 Tuc again showing the location of the CN-strong and CN-weak stars. These two 47 Tuc populations, as well as differing in carbon abundances, differ in nitrogen abundances by a factor of $\sim 10$ with the CN-strong stars having the higher $\mathrm{N}$ abundances (Cannon et al. 1998, Briley et al. $2004)$. The plot shows that below $[\mathrm{Fe} / \mathrm{H}] \approx-1.3$, we have little sensitivity to changes in the CN-band strength. This is a consequence of the moderate resolution of the spectra, the temperature of the stars, and the fact that the CN-band strength is proportional to the product of the $\mathrm{C}$ and $\mathrm{N}$ abundances, and so decreases much faster with $[\mathrm{Fe} / \mathrm{H}]$ than do $\mathrm{CH}$ (or NH) features. Nevertheless, the plot reveals a number of interesting features. First, the strong G-band stars identified in the left panel of Fig. 1 do not generally stand out from the bulk of the population in the right panel. However, for $[\mathrm{Fe} / \mathrm{H}] \geqslant-1.3$ where we have sensitivity to CN-band strength changes, it is possible that some of the strong G-band stars are $\mathrm{CN}$-weak, and that the most metal-rich example of these stars is $\mathrm{CN}$ strong. Second, there is clearly a population of $\omega$ Cen main sequence stars which do have strong, or in some cases extremely strong, CN-band strengths. These stars are plotted as filled triangles. Of the 32 stars in our sample with $[\mathrm{Fe} / \mathrm{H}] \geqslant-1.3,12$, or approximately $40 \%$, have strong cyanogen indices. Spectrum synthesis calculations show that these CNstrong stars have nitrogen enhancements of up to a factor of $\sim 100$ (the best fit for the 

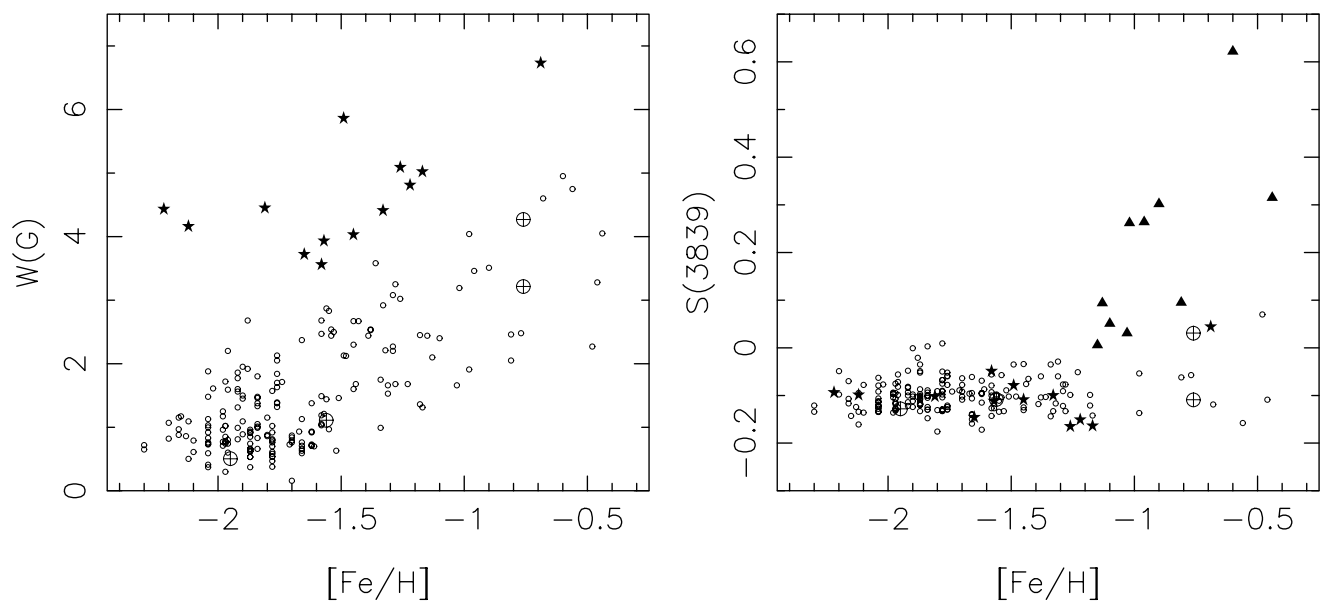

Figure 1. (a) Left panel: The strength of the G-band index $\mathrm{W}(\mathrm{G})$ is plotted against metallicity for the sample of $\omega$ Cen stars with $V>18$. Stars with large values of $W(G)$ for their abundance are plotted as filled stars. (b) Right panel: The strength of the cyanogen index $\mathrm{S}(3839)$ is plotted against metallicity for same $\omega$ Cen sample. The strong G-band stars from the left panel are again plotted as filled stars, while stars with large values of $\mathrm{S}(3839)$ for their abundance are plotted as filled triangles. In both panels the circled plus symbols are the mean locations for similar samples of main sequence stars in the globular clusters NGC 6397, NGC 6752 and 47 Tuc. For the latter cluster there are two points: the $\mathrm{CN}$-strong stars are $\mathrm{CH}$-weak and vice-versa.

star with the largest $\mathrm{S}(3839)$ value has $[\mathrm{C} / \mathrm{Fe}] \approx+0.2$ and $[\mathrm{N} / \mathrm{Fe}] \approx+2.0)$. In general, the best fits to the spectra of these $\mathrm{CN}$-strong stars do not require large carbon depletions (or enhancements).

\section{4. $\mathrm{SrII} 407 \mathrm{r}$}

In Fig. 2 we plot a measure of the strength of the SrII line at $4077 \AA$ against $[\mathrm{Fe} / \mathrm{H}]$ for our sample of $\omega$ Cen main sequence stars. Again the circled plus symbols represent the mean locations for the standard clusters, noting that for 47 Tuc there is no difference between the CN-strong and CN-weak stars as regards SrII line strengths. The $\omega$ Cen strong Gband and strong-CN stars are plotted with the same symbols as for Fig. 1. Given the resolution and $\mathrm{S} / \mathrm{N}$ of our spectra, the dispersion in the $\mathrm{Sr}(4077)$ values in Fig. 2 is largely the result of observational uncertainties, particularly at lower abundances. Equivalently, at this resolution and $\mathrm{S} / \mathrm{N}$, we are sensitive only to large (positive) variations in the $\mathrm{Sr}$ abundance. We concentrate on three stars that stand out in this figure, the strong G-band star at intermediate metallicities, the CN-strong star at the high end of the metallicity distribution, and a further metal-rich star that was previously undistinguished, i.e., its spectrum shows no evidence for significant $\mathrm{C}$ or $\mathrm{N}$ enhancements (or depletions). Indeed spectrum synthesis calculations for this star show that it may be mildly depleted in $\mathrm{C}$ $(\leqslant 0.3 \mathrm{dex})$ and that it has an apparently normal $\mathrm{N}$ abundance.

The spectra of these 3 stars do indeed point to substantial Sr overabundances. In each case not only is the SrII $4077 \AA$ line significantly enhanced but also the SrII line at $4215 \AA$ is unusually strong. We have conducted spectrum synthesis calculations of the region

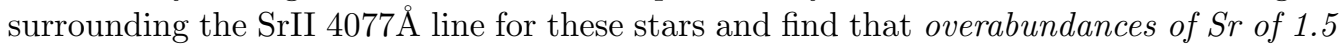
to $1.8 \mathrm{dex}$ are required to fit the line strengths. We have also investigated the spectra of these Sr-strong stars in the vicinity of the BaII line at $4554 \AA$. The synthetic spectra calculations show that in both the (strong G-band, Sr-strong) star and the (CN-strong, Sr-strong) star, the BaII line strengths are suggestive of Ba overabundances comparable 


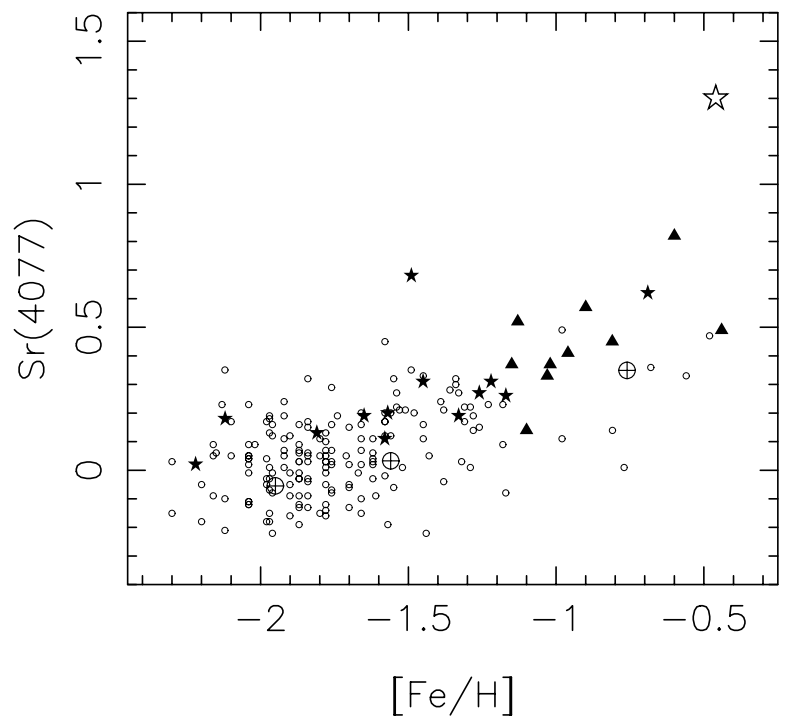

Figure 2. The strength of the SrII line at $4077 \AA$ is plotted against metallicity for the sample of $\omega$ Cen stars with $\mathrm{V}>18$. Symbols as for Fig. 1 except that the previously undistinguished star with a large SrII strength is plotted as an open star. The circled plus symbols are again the mean locations for NGC 6397, NGC 6752 and 47 Tuc.

to that seen for strontium. However, for the star without substantial C or N enhancements (or depletions), the BaII $4554 \AA$ line is barely visible, and Ba overabundances comparable to that seen for Sr can be definitely ruled out.

\section{Discussion}

As regards the $\mathrm{C}$ and $\mathrm{N}$ enhanced stars, it seems plausible that we are seeing the effects of 3rd dredge-up combined with mass loss from intermediate-mass thermally-pulsing AGB (TP-AGB) stars. The difference between whether a star is $\mathrm{C}$ or $\mathrm{N}$ enhanced can then be ascribed to whether or not hot-bottom burning occurred in the TP-AGB star to convert dredged-up carbon into nitrogen. What our data cannot answer, however, is whether these enhanced abundances are a surface effect, e.g. resulting from mass transfer in a binary, or are intrinsic abundances, i.e. the star formed from gas with these abundances. The question is not easily tackled. In the field, it is generally accepted that the majority of CH-stars (and BaII-stars) are indeed binary systems, with the observed atmospheric abundances resulting from wind-driven mass transfer from the original primary to the companion (now the observed star) during the primary's evolution through the TP-AGB phase (e.g. McClure 1984). This is certainly also a reasonable interpretation for the C-enhanced main sequence stars observed here, given that they are found at all $[\mathrm{Fe} / \mathrm{H}]$ values, that they have a relatively low frequency of occurrence, which is likely to be compatible with a reasonable original total binary fraction, and that the relatively low central concentration of $\omega$ Cen means that the appropriate binaries are not likely to have been disrupted before the original primary reaches the TP-AGB phase.

It is less obvious that this binary mass transfer explanation can also be applied for the N-rich stars. While we have no constraints on the number of such stars for abundances less than $[\mathrm{Fe} / \mathrm{H}] \approx-1.3$, above this abundance the number of N-rich stars is substantial ( $\sim 40 \%$ of the total). Interpreting all these stars as the result of mass transfer in binaries would seem to require an implausibly high original total binary fraction. The alternative 
is that these stars formed from material that was substantially enhanced in nitrogen. In this aspect, such a process may be related to that which drives the primordial abundance variations in other globular clusters, as that phenomenon is known to occur in $\omega$ Cen (Norris \& Da Costa 1995a). One potential method to investigate this question is to compare the frequency of N-enhanced relatively metal-rich giants with that observed in our main sequence sample. If the effect is primarily a mass-transfer driven atmospheric one, then the frequency of occurrence in red giants should be reduced relative to that in main sequence stars, as in at least some main sequence stars the current unusual surface abundances should be significantly diluted by convective mixing as the star evolves up the red giant branch. Conversely, if the effect is primarily primordial, the main sequence and RGB frequencies should be comparable, although the possibility that some stars could become N-enhanced through evolutionary mixing as they ascend the RGB must also be considered. The existing data for red giants do not provide any strong guidance. Norris \& Da Costa (1995b) list six stars with $[\mathrm{Fe} / \mathrm{H}] \geqslant-1.3$ for which they have determined $\mathrm{N}$ abundances. While noting the caveats expressed in that paper concerning selection biases and potential systematic errors in the $\mathrm{N}$ abundances, it is interesting that 3 of the 6 stars are N-enhanced, nominally a similar ratio to that for the main sequence. The size of the N-enhancements, however, typically a factor of 5-15 (Norris \& Da Costa 1995b), are apparently notably less than for the main sequence stars.

As regards the $\mathrm{Sr}$ overabundances, those associated with $\mathrm{C}$ and/or $\mathrm{N}$ enhancements can be ascribed to s-process nucleosynthesis occuring in the same TP-AGB stars that provide the carbon and/or nitrogen enhancements. This is particularly likely in those situations where $\mathrm{Ba}$ overabundances are also seen. What is much less clear is whether this mechanism could also apply to the sole metal-rich star in our main sequence sample which has a large overabundance of Sr, but which lacks significant enhancements (or depletions) in $\mathrm{C}$ and $\mathrm{N}$, and which does not have an overabundance of Ba matching that of strontium. A high dispersion spectrum of this star, to allow determination of the abundances of a number of other elements (e.g. $\alpha, \mathrm{O}, \mathrm{Na}, \mathrm{Al}, \mathrm{Mg}$ and r- and s-process), is required in order to understand its origin.

In summary, our moderate resolution spectra of a large sample of $\omega$ Cen main sequence stars have revealed a number of unusual objects. Some of these stars may be the result of mass transfer in binary systems, but others seem to require more exotic explanations. Further observational and theoretical studies are clearly necessary.

\section{References}

Beers, T.C., Rossi, S., Norris, J.E., Ryan, S.G., \& Shefler, T. 1999, AJ 117, 981

Bell, R.A., \& Dickens, R.J. 1974, MNRAS 166, 89

Briley, M.M., Harbeck, D., Smith, G.H., \& Grebel, E.K. 2004, AJ 127. 1588

Cannon, R.D., et al. 1998, MNRAS 298, 601

Hilker, M., Kayser, A., Richtler, T., \& Willemsen, P. 2004, A\&A 422, L9

Lloyd-Evans, T. 1983, MNRAS 204, 975

McClure, R.D. 1984, ApJ 280, L31

Norris, J.E., \& Da Costa, G.S. 1995a, ApJ 441, L81

Norris, J.E., \& Da Costa, G.S. 1995b, ApJ 447, 680

Norris, J.E., Freeman, K.C., Mayor, M., \& Seitzer, P. 1997, ApJ 487, L187

Pancino, E., Pasquini, L., Hill, V., Ferraro, F.R., \& Bellazzini, M. 2002, ApJ 568, L101

Rey, S.-C., Lee, Y.-W., Ree, C.H., Joo, M.-J., Sohn, Y.-J., \& Walker, A.R. 2004, AJ 127, 958

Smith, V.V., et al. 2000, AJ 119, 1239

Sollima, A., Ferraro, F.R., Pancino, E., \& Bellazzini, M. 2005, MNRAS 357, 265

Vanture, A.D., Wallerstein, G., \& Suntzeff, N. 2002, ApJ 569, 984 